\title{
KOREA-JAPAN-6
}

\section{Validation of difficulty scoring system for laparoscopic distal pancreatectomy}

\author{
$\underline{\text { Kohei NAKATA* }}^{*}$ \\ Department of Surgery and Oncology, Kyushu University, Fukuoka, Japan
}

Backgrounds: Laparoscopic distal pancreatectomy (LDP) is gaining acceptance as a standard approach for removal of various benign and malignant pancreatic diseases. There are several methods of LDP which includes classic LDP with splenectomy for benign diseases, laparoscopic spleen and vessel preserving distal pancreatectomy, and laparoscopic radical pancreatectomy for invasive pancreatic cancers for pancreatic cancer. Several conditions may contribute to the difficulty of LDP based on type of operation, characteristics of the disease, patients' body habitus and tumor location. We have previously developed a difficulty scoring (DS) system to quantify the degree of difficulty in LDP to aid surgeons who would like to learn or teach this procedure in a stepwise manner.

$<$ Aim>: The aim of this study is to validate the DS system in LDP, proposed by the study group of Japan Society for Hepato-Biliary-Pancreatic Surgery/Asian-Pacific Hepato-Pancreato-Biliary Association in 2018 (J Hepatobiliary Pancreato Sci 2018;25:489-497). Effect of score assessed by DS system on surgical outcomes including conversion rate, operation time and blood loss was validated in both Korean and Japanese cohort.

Results: We found significant difference of operation time and conversion rates in both Japanese and Korean cohort. However, the DS was not related with blood loss in both cohorts.

Conclusions: We observed associations between the DS system and operative outcomes, to some extent, and the trend seems to be same in both Korean and Japanese. To make the DS system for more useful one, we would like to modify the system in the future. 